\title{
ANALYTIC VARIETIES WITH FINITE VOLUME AMOEBAS ARE ALGEBRAIC
}

\author{
FARID MADANI AND MOUNIR NISSE
}

\begin{abstract}
In this paper, we study the amoeba volume of a given $k$-dimensional generic analytic variety $V$ of the complex algebraic torus $\left(\mathbb{C}^{*}\right)^{n}$. When $n \geq 2 k$, we show that $V$ is algebraic if and only if the volume of its amoeba is finite. In this precise case, we establish a comparison theorem for the volume of the amoeba and the coamoeba. Examples and applications to the $k$-linear spaces will be given.
\end{abstract}

\section{INTRODUCTION}

Some fundamental questions concerning the complex logarithm lead us to study certain mathematical objects called amoebas and coamoebas, which are natural projections of complex varieties. They have a strong relations to several other areas of mathematics as real algebraic geometry, tropical geometry, complex analysis, mirror symmetry, algebraic statistics and in several other areas. Amoebas degenerate to a piecewise-linear object called tropical varieties, (see [M1-02], [M2-04], [M3-00], [FPT-00], [NS-09], [PR-04, and [PS-04]). The behavior of a coamoeba at infinity is the so called the phase limit set of the variety itself. For a $k$-dimensional complex algebraic variety, the phase limit set contains an arrangement of $k$-dimensional flat torus, which plays a crucial role on the geometry and the topology of both amoeba and coamoeba. Moreover, these objects are used as an intermediate link between the classical and the tropical geometry. In this paper, we underline that the geometry of the amoeba affects the algebraic structure of the variety.

It was shown by Passare and Rullgård [PR-04 that the area of complex algebraic plane curve amoebas are finite. In [MN-11], we proved that the volume of the amoeba of a generic $k$-dimensional algebraic variety of the complex algebraic torus $\left(\mathbb{C}^{*}\right)^{n}$ with $n \geq 2 k$, is finite. There now arises the reciprocal question: Let $\mathcal{I}$ be an ideal in the ring of holomorphic function on $\mathbb{C}^{n}$, and $\bar{V}$ be the set of zeros of $\mathcal{I}$ which we assume generic of dimension $k \leq \frac{n}{2}$. If we assume that the volume of the amoeba of $V=\bar{V} \cap\left(\mathbb{C}^{*}\right)^{n}$ is finite, then, is $V$ algebraic? Theorem 1.1 gives an affirmative answer to this question.

1991 Mathematics Subject Classification. 14T05, 32A60.

Key words and phrases. Analytic varieties, algebraic varieties, amoebas, coamoebas, logarithmic limit sets, phase limit sets, spherical polyhedrons.

The first author was supported by Alexander von Humboldt Foundation and by the Mathematisches Forschungsinstitut Oberwolfach in 2011, through the program "Oberwolfach Leibniz Fellows.

Research of the second author is partially supported by NSF MCS grant DMS-0915245. 
The Main theorem of this paper is the following:

Theorem 1.1. Let $V$ be a generic $k$-dimensional analytic variety in $\left(\mathbb{C}^{*}\right)^{n}$ with $n \geq 2 k$. The following assertions are equivalent:

(i) The variety $V$ is algebraic;

(ii) The volume of $\mathscr{A}(V)$ is finite.

This paper is organized as follows. In Section 4, using the geometry and the combinatorial structure of the logarithmic limit and the phase limit sets, we prove Theorem 1.1 in the special case of curves. The proof of this case is actually the crucial step to show the general one. In Section 5 , we show Theorem 1.1 for varieties of higher dimensions. In Section 6, we give some examples of plane and spatial complex curves, underlying the importance of the finiteness of their amoeba areas. In Section 3, we prove a comparison theorem, asserting that up to rational number, the amoeba volume is bounded above and below by the coamoeba volume. Finally, in Section 7 , we give an application of the comparison theorem to the $k$-dimensional affine linear spaces in $\left(\mathbb{C}^{*}\right)^{2 k}$. We compute the amoeba volumes of $k$-dimensional real affine linear spaces in $\left(\mathbb{C}^{*}\right)^{2 k}$.

\section{Preliminaries}

Let $W$ be a complex variety in $\mathbb{C}^{n}$ defined by an ideal $\mathcal{I}$ of holomorphic functions on $\mathbb{C}^{n}$. We say that a subvariety $V$ of the complex algebraic torus $\left(\mathbb{C}^{*}\right)^{n}$ is analytic if there exists a complex variety $W$ as above such that $V:=W \cap\left(\mathbb{C}^{*}\right)^{n}$. All the analytic varieties considered in this paper are defined as above. The amoeba $\mathscr{A}$ of $V$ is by definition (see M. Gelfand, M.M. Kapranov and A.V. Zelevinsky [GKZ-94]) the image of $V$ under the map :

$$
\begin{aligned}
\log :\left(\mathbb{C}^{*}\right)^{n} & \longrightarrow \mathbb{R}^{n} \\
\left(z_{1}, \ldots, z_{n}\right) & \longmapsto\left(\log \left|z_{1}\right|, \ldots, \log \left|z_{n}\right|\right) .
\end{aligned}
$$

It is well known that the amoeba of a variety of codimension one is closed and its complement components in $\mathbb{R}^{n}$ are convex (see [FPT-00] ). A. Henriques gives an analogous definition for the convexity of amoeba complements of higher codimension varieties as follows:

Definition 2.1. A subset $A \subset \mathbb{R}^{n}$ is called l-convex if for any l-plane $L \subset \mathbb{R}^{n}$ the induced homomorphism $H_{l-1}(L \backslash A) \longrightarrow H_{l-1}\left(\mathbb{R}^{n} \backslash A\right)$ is injective.

Also, he proves that if $V$ is a variety of codimension $l$ and $L$ is an $l$-plane of rational slope and $c$ is a non-zero $(l-1)$-cycle in $H_{l-1}(L \backslash \mathscr{A})$ then its image in $H_{l-1}\left(\mathbb{R}^{n} \backslash \mathscr{A}\right)$ is non-zero, and then $\mathbb{R}^{n} \backslash \mathscr{A}$ is l-convex (see [H-03] Theorem 4.1).

Let $V$ be an analytic variety as above. We denote by $\mathscr{L}^{\infty}(V)$ its logarithmic limit set which is the boundary of the closure of $r(\mathscr{A}(V))$ in the $n$-dimenstional ball $B^{n}$, where $r$ is the map defined by (see Bergman [B-71]):

$$
\begin{aligned}
r: \mathbb{R}^{n} & \longrightarrow B^{n} \\
x & \longmapsto r(x)=\frac{x}{1+|x|} .
\end{aligned}
$$

If $V$ is an algebraic variety of dimension $k$, then its logarithmic limit set is a finite rational spherical polyhedron of dimension $k-1$ (i.e., a finite union 
of finite intersections of closed hemispheres and can be described in terms of a finite number of inequalities with integral coefficients). More precisely, we have the following theorem structure (see [B-71] and [BG-84]):

Theorem 2.1 (Bergman, Bieri-Groves). The logarithmic limit set $\mathscr{L}^{\infty}(V)$ of an algebraic variety $V$ in $\left(\mathbb{C}^{*}\right)^{n}$ is a finite union of rational spherical polyhedrons. The maximal dimension of a polyhedron in this union is achieved at least by one polyhedron $P$ in this union, and we have $\operatorname{dim}_{\mathbb{R}} \mathscr{L}^{\infty}(V)=$ $\operatorname{dim}_{\mathbb{R}} P=\left(\operatorname{dim}_{\mathbb{C}} V\right)-1$.

The argument map is the map defined as follows:

$$
\text { Arg : } \begin{array}{cl}
\left(\mathbb{C}^{*}\right)^{n} & \longrightarrow\left(S^{1}\right)^{n} \\
\left(z_{1}, \ldots, z_{n}\right) & \longmapsto\left(\arg \left(z_{1}\right), \ldots, \arg \left(z_{n}\right)\right) .
\end{array}
$$

where $\arg \left(z_{j}\right)=\frac{z_{j}}{\left|z_{j}\right|}$. The coamoeba of $V$, denoted by coA , is its image under the argument map (defined for the first time by Passare on 2004). On 2009, Sottile and the second author [NS-09] define the phase limit set of $V$, $\mathscr{P}^{\infty}(V)$, as the set of accumulation points of arguments of sequences in $V$ with unbounded logarithm. If $V$ is an algebraic variety of dimension $k$, $\mathscr{P} \infty(V)$ contains an arrangement of $k$-dimensional real sub-torus.

\section{Comparison Between Amoeba and Coamoeba volumes}

For a given map $f$, we denote by $\operatorname{Jac}(f)$ the Jacobian matrix of $f$, and by $\mathrm{J}(f)$ the determinant of $\operatorname{Jac}(f)$ when it exists.

Proposition 3.1. Let $V$ be a $k$-dimensional complex submanifold in $\left(\mathbb{C}^{*}\right)^{n}$. The maps Log and Arg are well defined on $V$ and

$$
\partial \log =\frac{1}{\operatorname{Arg}} \partial \operatorname{Arg}, \quad \bar{\partial} \log =\frac{-1}{\operatorname{Arg}} \bar{\partial} \operatorname{Arg}, \quad\left|J\left(\log _{I}\right)\right|=\left|J\left(\operatorname{Arg}_{I}\right)\right|,
$$

for any $I \subset\{1, \ldots, n\}$ with cardinal $2 k$.

Proof. We denote by $\left\{z_{j}\right\}_{1 \leq j \leq n}$ the complex coordinates on $\mathbb{C}^{n}$ and by $\left\{t_{j}\right\}_{1 \leq j \leq k}$ the complex coordinates on $V$ given by a local chart $(\Omega, f)$ (i.e. $\left.\forall z \in \Omega, t_{j}=f_{j}(z)\right)$, where $\Omega$ is an open set of $V$ and $f$ is holomorphic from an open set of $\mathbb{C}^{n}$ to $\mathbb{C}^{k}$. Since $V$ is a complex submanifold of $\left(\mathbb{C}^{*}\right)^{n}$, the injection map $\imath: V \hookrightarrow\left(\mathbb{C}^{*}\right)^{n}$ is holomorphic. By definition, for any $z \in V$ we have $\imath(z)=e^{\log z} \operatorname{Arg} z$. Since $\imath$ is holomorphic, $\bar{\partial} \imath(z)=0$ for any $z \in V$ (i.e. $\left.\forall j \leq k, \partial_{\bar{t}_{j}} \imath(z)=0\right)$ ). It implies that for any $j=1, \ldots, k$ and $z \in \Omega$

$$
\partial_{\bar{t}_{j}} \log (z)=-\frac{1}{\operatorname{Arg}(z)} \partial_{\bar{t}_{j}} \operatorname{Arg}(z), \quad \partial_{t_{j}} \log (z)=\frac{1}{\operatorname{Arg}(z)} \partial_{t_{j}} \operatorname{Arg}(z) .
$$

where the second equality holds by conjugating the first one. The statement of the proposition follows.

Theorem 3.1. Let $V$ be an analytic variety of $\left(\mathbb{C}^{*}\right)^{n}$ of dimension $k \leq \frac{n}{2}$. Let $\mathscr{A}$, cod be the amoeba and coamoeba of $V$ respectively. We suppose that $\log : V \rightarrow \mathscr{A}$ and $\operatorname{Arg}: V \rightarrow$ cod are locally finite coverings. We define two rational numbers

$$
p=\frac{\min _{\theta \in \operatorname{co} \backslash \backslash \operatorname{Arg} S} \# \operatorname{Arg}^{-1}\{\theta\}}{m_{y \in \mathscr{A} \backslash \log S} \# \log ^{-1}\{y\}}, \quad P=\frac{\max _{\theta \in \operatorname{co} \mathscr{A} \backslash \operatorname{Arg} S} \# \operatorname{Arg}^{-1}\{\theta\}}{\min _{y \in \mathscr{A} \backslash \log S} \# \log ^{-1}\{y\}} .
$$


Then

$$
p \operatorname{vol}(\operatorname{co\mathscr {A}}) \leq \operatorname{vol}(\mathscr{A}) \leq P \operatorname{vol}(\operatorname{co\mathscr {A}}) .
$$

In particular the volume of $\mathscr{A}$ is finite.

As an application of Theorem 3.1. we have the following result that the authors had already proven in $\mathrm{MN}-11$ :

Corollary 3.1. The amoeba of a $k$-dimensional generic complex algebraic variety in $\left(\mathbb{C}^{*}\right)^{n}$, with $2 k \leq n$ has a finite volume.

Proof. If $V$ is a generic complex algebraic variety in $\left(\mathbb{C}^{*}\right)^{n}$ of dimension $k \leq \frac{n}{2}$, then $\log : V \rightarrow \mathscr{A}$ and $\operatorname{Arg}: V \rightarrow \operatorname{co} \mathscr{A}$ are locally finite coverings. Therefore, the statement follows from Theorem 3.1

Proof of Theorem 3.1. We have $V=V_{\text {reg }} \cup V_{\text {sing }}$ where $V_{\text {reg }}$ is the regular part of $V$ which is a $k$-dimensional complex submanifold in $\mathbb{C}^{n}$ and $V_{\text {sing }}$ is the singular part of $V$ which is a analytic subset of pure dimension less or equal than $k-1$. By Sard's theorem, the $2 k$-measure of $\log \left(V_{\text {sing }}\right)$ and $\operatorname{Arg}\left(V_{\text {sing }}\right)$ is zero. Hence, without loss of generality, we can suppose that $V=V_{\text {reg }}$ is a $k$-dimensional complex submanifold endowed with the induced metric $\imath^{*} \mathcal{E}_{2 n}$, where $\mathcal{E}_{2 n}$ is the standard Euclidean metric on $\mathbb{C}^{n}$ and $\imath: V \rightarrow \mathbb{C}^{n}$ is the injection map. Let

$$
S=\left\{z \in V \mid \operatorname{rank} \operatorname{Jac}_{\log }<2 k\right\}
$$

be the set of critical points of Log. Using Proposition 3.1, we conclude that the Jacobian matrix of $\log$ and Arg have the same rank at any point in $V$. Hence, the set of critical points of Arg is also $S$. Using again Sard's theorem, the $2 k$-measure of $\log S$ and $\operatorname{Arg} S$ is zero. It yields $\operatorname{vol}(\mathscr{A})=\operatorname{vol}(\mathscr{A} \backslash \log S)$

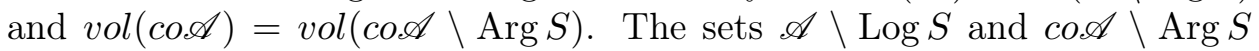
are $2 k$-dimensional real immersed submanifolds of $\mathbb{R}^{n}$ and $\mathbb{T}^{n}$ respectively. They are endowed with the induced metric $\imath^{*} \mathcal{E}_{n}$. Let $U_{1}$ and $U_{2}$ be two open sets in $V \backslash S$ such that $\log : U_{1} \rightarrow \mathscr{A} \backslash \log S$ and $\operatorname{Arg}: U_{2} \rightarrow \operatorname{co\mathscr {A}} \backslash \operatorname{Arg} S$ are diffeomorphisms. By construction, we have the following identities:

$$
\begin{aligned}
& \operatorname{vol}(\mathscr{A})=\operatorname{vol}\left(\mathscr{A} \backslash \log S, \imath^{*} \mathcal{E}_{n}\right)=\operatorname{vol}\left(U_{1}, \log { }^{*} \mathcal{E}_{n}\right),
\end{aligned}
$$

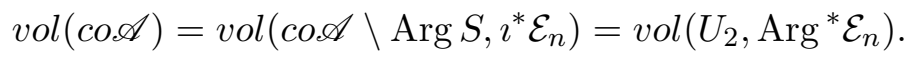

Following the construction in MN-11] (see Section 3) and the identities above, the volume of $\mathscr{A}$ and $\operatorname{co} \mathscr{A}$ are given by

$$
\begin{aligned}
& \operatorname{vol}(\mathscr{A})=\operatorname{vol}\left(U_{1}, \log { }^{*} \mathcal{E}_{n}\right)=\int_{U_{1}}\left|\psi_{2 k}\right|_{\log * \mathcal{E}_{n}} \mathrm{~d} v, \\
& \operatorname{vol}(\operatorname{co\mathscr {A}})=\operatorname{vol}\left(U_{2}, \operatorname{Arg}{ }^{*} \mathcal{E}_{n}\right)=\int_{U_{2}}\left|\psi_{2 k}\right|_{\operatorname{Arg} * \mathcal{E}_{n}} \mathrm{~d} v
\end{aligned}
$$

where $\mathrm{d} v$ is the restriction of the volume form of $\mathbb{C}^{n}$ to $V$, and $\psi_{2 k}$ is $2 k$-vector field in $\Lambda^{2 k} T V$, such that $\mathrm{d} v\left(\psi_{2 k}\right)=1$. In a local complex coordinates $\left\{t_{j}\right\}_{1 \leq j \leq k}$, the volume form $\mathrm{d} v$ and $\psi_{2 k}$ are given by

$$
\mathrm{d} v=i^{k} \mathrm{~d} t \wedge \mathrm{d} \bar{t}, \quad \psi_{2 k}=(-i)^{k} \frac{\partial}{\partial t} \wedge \frac{\partial}{\partial \bar{t}}
$$


where $\mathrm{d} t=\mathrm{d} t_{1} \wedge \cdots \wedge \mathrm{d} t_{k}$ and $\frac{\partial}{\partial t}=\frac{\partial}{\partial t_{1}} \wedge \cdots \wedge \frac{\partial}{\partial t_{k}}$. It yields that

$$
\begin{aligned}
\left|\psi_{2 k}\right|_{\log *^{*} \mathcal{E}_{n}}^{2} & =\left|\frac{\partial \log }{\partial t} \wedge \frac{\partial \log }{\partial \bar{t}}\right|_{\mathcal{E}_{n}}^{2}=\sum_{I \subset\{1, \ldots, n\}, \# I=2 k}|J(\log I)|^{2} \\
\left|\psi_{2 k}\right|_{\operatorname{Arg} *^{*} \mathcal{E}_{n}}^{2} & =\left|\frac{\partial \operatorname{Arg}}{\partial t} \wedge \frac{\partial \operatorname{Arg}}{\partial \bar{t}}\right|_{\mathcal{E}_{n}}^{2}=\sum_{I \subset\{1, \ldots, n\}, \# I=2 k}|J(\operatorname{Arg} I)|^{2}
\end{aligned}
$$

where, $\log _{I}(z)=\left(\log \left|z_{i_{1}}\right|, \ldots, \log \left|z_{i_{2 k}}\right|\right)$ for all $z \in V$ and $I=\left\{i_{1}, \ldots, i_{2 k}\right\}$, and $\mathrm{J}\left(\log _{I}\right)$ is the Jacobian determinant of $\log I$ with respect to $\left\{t_{j}, \bar{t}_{j}\right\}_{1 \leq j \leq k}$. We have from Proposition 3.1, $\left|J\left(\log _{I}\right)\right|=\left|J\left(\operatorname{Arg}_{I}\right)\right|$ for any $I \subset\{1, \ldots, n\}$ with cardinal $2 k$. We deduce that

$$
\left|\psi_{2 k}\right|_{\log * \mathcal{E}_{n}}=\left|\psi_{2 k}\right|_{\operatorname{Arg} * \mathcal{E}_{n}} .
$$

It remains to compare between the volume of $\mathscr{A}$ and $\cos$, given by (1), (2). Since $\log : V \rightarrow \mathscr{A}$ and Arg $: V \rightarrow \operatorname{co} \mathscr{A}$ are locally finite coverings,

$$
\begin{gathered}
\frac{1}{M_{1}} \int_{V \backslash S}\left|\psi_{2 k}\right|_{\log * \mathcal{E}_{n}} \mathrm{~d} v \leq \operatorname{vol}(\mathscr{A}) \leq \frac{1}{m_{1}} \int_{V \backslash S}\left|\psi_{2 k}\right|_{\log * \mathcal{E}_{n}} \mathrm{~d} v, \\
\frac{1}{M_{2}} \int_{V \backslash S}\left|\psi_{2 k}\right|_{\operatorname{Arg} * \mathcal{E}_{n}} \mathrm{~d} v \leq \operatorname{vol}(\cos ) \leq \frac{1}{m_{2}} \int_{V \backslash S}\left|\psi_{2 k}\right|_{\operatorname{Arg} * \mathcal{E}_{n}} \mathrm{~d} v,
\end{gathered}
$$

where $M_{1}=\max _{y \in \mathscr{A} \backslash \log S} \# \log ^{-1}\{y\}, m_{1}=\min _{y \in \mathscr{A} \backslash \log S} \# \log ^{-1}\{y\}$, $M_{2}=\max _{\theta \in \mathscr{A} \backslash \operatorname{Arg} S} \# \operatorname{Arg}^{-1}\{\theta\}$ and $m_{2}=\min _{\theta \in \mathscr{A} \backslash \operatorname{Arg} S} \# \operatorname{Arg}^{-1}\{\theta\}$. We conclude that

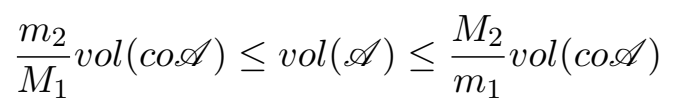

\section{Analytic Curves with finite area amoebas are algebraic}

The purpose of this section is to prove the following:

Theorem 4.1. Let $\mathscr{C} \subset\left(\mathbb{C}^{*}\right)^{n}$ be an analytic curve defined by an ideal of holomorphic functions $\mathcal{I}(\mathscr{C})$ with $n \geq 2$. The amoeba area of $\mathscr{C}$ is finite if and only if $\mathscr{C}$ is algebraic.

In order to prove Theorem 4.1, we start by proving a series of three lemmas.

Lemma 4.1. Let $\mathscr{C}$ be a curve as in Theorem 4.1. The logarithmic limit set $\mathscr{L}^{\infty}(\mathscr{C})$ has the following properties:

(i) Each point $s \in \mathscr{L}^{\infty}(\mathscr{C})$ has a rational slope (i.e., there exists $\lambda \in \mathbb{R}$ such that $\left.\lambda \cdot \overrightarrow{O s} \in \mathbb{Z}^{n}\right)$;

(ii) The logarithmic limit set $\mathscr{L}^{\infty}(\mathscr{C})$ is a finite set.

Proof. (i) Assume on the contrary that $\mathscr{L}^{\infty}(\mathscr{C})$ contains a point $s$ such that the vector $\overrightarrow{O s}$ has an irrational slope. Let $s=\left(u_{1}, \ldots, u_{n}\right)$, and

$$
\begin{aligned}
\mathbb{R} & \longrightarrow \mathbb{R}^{n} \\
x & \longmapsto\left(u_{1} x+a_{1}, \ldots, u_{n} x+a_{n}\right)
\end{aligned}
$$


be the parametrization of the asymptotic straight line $D_{s}$ in $\mathbb{R}^{n}$ to the amoeba $\mathscr{A}(\mathscr{C})$, in direction $s$. Under this assumption, the phase limit set $\mathscr{P} \infty(\mathscr{C})$ contains a subset of dimension at least 2 . Indeed, there exists an affine line in the universal covering of the real torus $\left(S^{1}\right)^{n}$, parametrized by $y \mapsto\left(u_{1} y_{1}+b_{1}, \ldots, u_{n} y_{n}+b_{n}\right)$, such that the closure of its projection in $\left(S^{1}\right)^{n}$ is a 2 -dimensional torus $T_{s}$, and is contained in the phase limit set $\mathscr{P} \infty(\mathscr{C})$. It implies that there exists a regular open subset $U \subset \operatorname{co\mathscr {A}}(\mathscr{C})$ which is covered infinitely many times under the argument map. Namely, $\operatorname{Arg}^{-1}(U)=\cup_{i=1}^{\infty} V_{i}$, where $V_{i}$ are open, regular and disjoint sets in $\mathscr{C}$. In

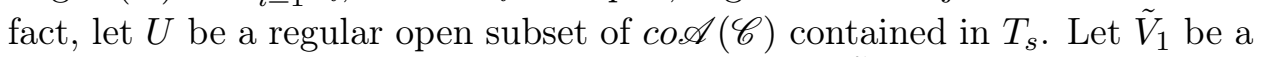
connected component of $\operatorname{Arg}^{-1}(U)$ and $V_{1}=\log \left(\tilde{V}_{1}\right)$. Let $I_{2}$ be a segment in $D_{s} \backslash \bar{V}_{1}$ such that $\operatorname{Arg}\left(\log ^{-1}\left(I_{2}\right)\right)$ intersect $U$. Indeed, $I_{2}$ exists because the immersed circle of slope $s$ in the real torus $\left(S^{1}\right)^{n}$ is dense in $T_{s}$. Let $\tilde{V}_{2}$ be the connected component of $\operatorname{Arg}\left(\log ^{-1}(U)\right)$ such that $\log \left(\tilde{V}_{2}\right)$ contains $I_{2}$, and $V_{2}=\log \left(\tilde{V}_{2}\right)$. By construction, $V_{1} \cap V_{2}$ is empty. We do the same thing for $V_{2}$, we obtain then an infinite sequence of open subsets $V_{1}, V_{2}, \ldots$ in the amoeba such that: $V_{i} \cap V_{j}=\phi$ for each $i \neq j$, and the area of $V_{i}$ is equal to the area of $U$ for each $i$ (this comes from the fact that the absolute value of the determinant of the jacobian of $\log \circ \mathrm{Arg}^{-1}$ is equal to one, which means that locally the map $\log \circ \mathrm{Arg}^{-1}$ conserves the volume). This is a contradiction with the finiteness of the area of the amoeba. Hence, $s$ cannot be irrational.

(ii) If $\mathscr{L}^{\infty}(\mathscr{C})$ is not finite, then it contains an accumulation point, since $S^{n-1}$ is compact. Let $s \in \mathscr{L}^{\infty}(\mathscr{C})$ be an accumulation point. Namely, there exists a sequence of points $s_{m} \in \mathscr{L}^{\infty}(\mathscr{C})$ such that $\lim _{m \rightarrow+\infty} s_{m}=s$, where each $s_{m}$ has a rational slope, and up to a multiplication by a real number, $\overrightarrow{O s}_{m}=\left(u_{1}^{(m)}, \ldots, u_{n}^{(m)}\right)$, with $u_{j}^{(m)}=\frac{a_{j}^{(m)}}{b_{j}^{(m)}}$, where $a_{j}^{(m)}$ and $b_{j}^{(m)}$ are integers. The sequence $\left\{s_{m}\right\}$ converges and is not stationary. This means that there exists $1 \leq j \leq n$ such that the sequence $\left\{b_{j}^{(m)}\right\}$ is unbounded. The circles corresponding to that slopes, have length greater or equal to $2 \pi b_{j}^{(m)}$. Hence, if $U$ is a regular subset of measure different than zero in the coamoeba, then it is covered infinitely many times under the argument map since the $b_{j}^{(m)}$ tend to infinity. This fact contradicts the fact that the area of the amoeba is finite. So, $\mathscr{L}^{\infty}(\mathscr{C})$ is finite.

Lemma 4.2. Let $\mathscr{C} \subset\left(\mathbb{C}^{*}\right)^{n}$ be a generic analytic curve such that the area of its amoeba is finite, and $s$ be a point in $\mathscr{L}^{\infty}(\mathscr{C})$. The number of ends $\mathscr{E}$ of the amoeba $\mathscr{A}(\mathscr{C})$, such that $\overline{r(\mathscr{E})} \cap S^{n-1}=\{s\}$, is finite.

Proof. Assume on the contrary that the number of ends $\mathscr{E}$ of the amoeba $\mathscr{A}(\mathscr{C})$, such that $\overline{r(\mathscr{E})} \cap S^{n-1}=\{s\}$, is infinite. Hence, two cases can hold. The first case: the number of corresponding circles of $s$ in $\left(S^{1}\right)^{n}$ is finite. Then, there exists at least a circle $C$ covered by the argument mapping infinitely many times. Take a regular open $U \subset \operatorname{coA}(\mathscr{C})$ bounded by a segment in $C$. Then, the number of connected components of $\operatorname{Arg}^{-1}(U)$ is infinite, and $\log \left(\operatorname{Arg}^{-1}(U)\right)$ has also an infinite number of connected 
components with the same area as $U$. This is a contradiction with the assumption.

The second case: the number of corresponding circles of $s$ in $\left(S^{1}\right)^{n}$ is infinite. Then, there exists a circle $C$ which is an accumulation circle with the same slope $s$, because the real torus is compact. Using the same reasoning as above, we obtain a contradiction.

This implies that the number of ends of the amoeba with the same slope is finite.

Lemma 4.3. Let $\mathscr{C}$ be a generic curve in $\left(\mathbb{C}^{*}\right)^{n}$. If $S^{n-2}$ is a sub-sphere of $S^{n-1}=\partial B^{n}$, invariant under the involution $-\mathrm{id}$, then $\mathscr{L}^{\infty}(\mathscr{C})$ intersects the interior of each connected components of $S^{n-1} \backslash S^{n-2}$.

Proof. Using Henriques theorem [H-03], we know that the complement components of amoebas are $l$-convex, if $\operatorname{codim} V=l+1$. Lemma 4.3 is a consequence of this fact. If $\mathscr{C}$ is generic (it is not contained in a complex algebraic torus of smaller dimension), then the intersection of the closed half spaces in $\mathbb{R}^{n}$ bounded by the hyperplanes normal to the directions $s \in \mathscr{L}^{\infty}(\mathscr{C})$ is compact.

Let $\mathscr{C} \subset\left(\mathbb{C}^{*}\right)^{n}$ be a generic analytic curve with defining ideal $\mathcal{I}(\mathscr{C})$ such that the area of its amoeba is finite. Let $s \in \mathscr{L}^{\infty}(\mathscr{C})$ with slope $\left(u_{1}, \ldots, u_{n}\right)$, and $D_{s}$ be a straight line in $\mathbb{R}^{n}$ directed by $s$ and asymptotic to the amoeba $\mathscr{A}$. We denote by $\mathscr{H}\left(D_{s}\right)$ the holomorphic cylinder which is the lifting of $D_{s}$ and asymptotic to the end of $\mathscr{C}$ corresponding to $s$.

Lemma 4.4. Let $\mathscr{C} \subset\left(\mathbb{C}^{*}\right)^{n}$ be a generic analytic curve as above. Then, the ideal $\mathcal{I}(\mathscr{C})$ is generated by a set of holomorphic functions $\left\{f_{1}, \ldots, f_{q}\right\}$ such that for any $j=1, \ldots, q$, we have $f_{j}=h_{j} g_{j}$, where $h_{j}$ are holomorphic functions without zeros in a neighborhood of $\mathscr{H}\left(D_{s}\right)$, and $g_{j}$ are entire functions where the terms of their power series have powers in the closed half space $\left\{\alpha \in \mathbb{R}^{n} \mid \sum_{i=1}^{n} \alpha_{i} \cdot u_{i} \leq 0\right\}$.

Proof. Without loss of generality, by Lemma 4.2, we can assume that the amoeba contains only one end corresponding to $s \in \mathscr{L}^{\infty}(\mathscr{C})$. If we don't have such decomposition of the $f_{j}$ 's, then $s$ is an accumulation point, and this cannot happen because the area of the amoeba is finite by assumption. So, there exist $h_{j}, g_{j}$, and $U(s)$ is a neighborhood of a holomorphic cylinder on which $h_{j}$ don't vanish. Namely, the holomorphic cylinder is the lifting in $\left(\mathbb{C}^{*}\right)^{n}$ via the logarithmic map of a straight line in $\mathbb{R}^{n}$ of slope $\left(u_{1}, \ldots, u_{n}\right)$. This holomorphic cylinder is such that the closure in $B^{n}$ of the retraction by $r$ (defined in Section 2) of the image under the logarithmic map of one of its ends, intersects the boundary of $B^{n}$ precisely on $s$. The function $g_{j}$ are entire where all their terms (as power series) are of powers $\alpha$ with $\sum u_{i} \alpha_{i} \leq 0$. Without loss of generality, we can assume that $\mathscr{C}$ is an irreducible curve. Hence, $\mathscr{C}$ is contained in the zero locus of the $g_{j}$ 's. In other words, our curve $\mathscr{C}$ is contained in the curve with defining ideal $\mathcal{I}_{s}$ spanned the by $g_{j}$ 's.

Proof of Theorem 4.1. If the area of the amoeba of $\mathscr{C}$ is finite, then using Lemma 4.3, and doing the same thing for each vertex in $\mathscr{L}^{\infty}(\mathscr{C})$ as in Lemma 4.4 we obtain polynomials $p_{1}, \ldots, p_{q}$ such that $\mathscr{C}$ is contained in $\mathscr{C}_{r}$ where 
$\mathscr{C}_{r}$ is the algebraic curve defined by the ideal generated by $p_{1}, \ldots, p_{q}$. Hence, $\mathscr{C}$ is an irreducible component of $\mathscr{C}_{r}$, then it is algebraic. If the curve is algebraic then using Corollary 3.1, we deduce that the area of the amoeba $\mathscr{C}$ is finite because the area of the coamoeba is always finite.

Corollary 4.1. Let $\mathscr{C}$ be an analytic curve (not necessary algebraic) in $\left(\mathbb{C}^{*}\right)^{n}$. Then $\mathscr{L}^{\infty}(\mathscr{C})$ is the union of a finite number of isolated points with rational slopes and a finite number of a geodesic arcs with rational end slopes. In particular, if $\mathscr{C}$ is not algebraic, then the number of arcs in $\mathscr{L}^{\infty}(\mathscr{C})$ is different than zero.

This is a consequence of the proof of Lemma 4.1. The arcs are geodesic (i.e., contained in some circle invariant under the involution $x \mapsto-x$ ) because the contrary means that the phase limit set contains a flat torus of dimension at least three, and this contradict the fact that the dimension of the coamoeba is equal two.

Corollary 4.2. Let $f$ be an entire function in two variables. Then $f=$ $h p$ where $h$ is a holomorphic function which doesn't vanish in the complex algebraic torus and $p$ is a polynomial if and only if the area of amoeba of the holomorphic curve in $\left(\mathbb{C}^{*}\right)^{2}$ defined by $f$ is finite.

\section{Proof of the Main theorem}

In this section, we generalize the result of Section 4 to k-dimensional analytic varieties in the complex algebraic torus $\left(\mathbb{C}^{*}\right)^{n}$ with $n \geq 2 k$. The following lemma generalizes Lemma 4.1 to higher dimensions.

Proposition 5.1. Let $V$ be a $k$-dimensional generic analytic variety in $\left(\mathbb{C}^{*}\right)^{n}$, with $n \geq 2 k$. Assume that the volume of its amoeba is finite. Then its logarithmic limit set $\mathscr{L}^{\infty}(V)$ is a finite rational spherical polyhedron of dimension $k-1$.

Proof. If $\mathscr{L}^{\infty}(V)$ is not a rational spherical polyhedron, and $v$ is a vertex of $\mathscr{L}^{\infty}(V)$ with irrational slope $u$, then the phase limit set $\mathscr{P}^{\infty}(V)$ contains a torus of dimension at least $(k-1)+2=k+1$ which is the closure of an immersed circle of slope $u$. Using the same argument as in the curve case (i.e., the fact that locally $\left|\mathrm{J}\left(\log \circ \operatorname{Arg}^{-1}\right)\right|=1$, the map $\log \circ \operatorname{Arg}^{-1}$ conserves the volumes), we deduce that the volume of $\mathscr{A}(V)$ in infinite. Indeed, if $U$ is a regular subset of the coamoeba $\operatorname{co} \mathscr{A}(V)$ with nonvanishing volume, then it is covered via the argument map infinitely many times. This is in contradiction with the assumption on the volume of the amoeba $\mathscr{A}(V)$. Therefore, $\mathscr{L}^{\infty}(V)$ is rational.

Now, suppose that $\operatorname{dim}\left(\mathscr{L}^{\infty}(V)\right)>k-1$. Then, it contains a point of irrational slope in some $l-$ cell with $l \geq k$. Hence, its phase limit set contains a torus of dimension at least $l+1 \geq k+1$. By the same argument as in the proof of Lemma 4.1, we obtain a contradiction on the assumption on the volume of the amoeba.

It remains to show that $\mathscr{L}^{\infty}(V)$ is finite. Otherwise, it contains an accumulation $(k-1)$-cell, which means that $\mathscr{P}^{\infty}(V)$ contains a torus of dimension 
at least $k+1$ (where $k+1=(k-1)+1+1$, such that $k-1$ comes from the dimension of the cells, the first one comes from the circle corresponding to the direction $s$, and the second one comes from the accumulation direction). Using the same reasoning as in Lemma 4.1 (ii), we get a contradiction on the assumption of the amoeba volume.

Definition 5.1. Let $s$ be a vertex of $\mathscr{L}^{\infty}(V)$. A polyhedron $P$ is in the direction of $s$, if for any point $x \in P$, there exists a vector $v_{x}$ in $P$ with starting point $x$ and slope $s$.

The following lemmas are the analogous of Lemma 4.2 and Lemma 4.4 in higher dimension and their proofs are similar.

Lemma 5.1. Let $V \subset\left(\mathbb{C}^{*}\right)^{n}$ be a generic $k$-dimensional analytic variety with defining ideal $\mathcal{I}(V)$ such that $n \geq 2 k$, and the volume of its amoeba is finite. Let $s \in \operatorname{Vert}\left(\mathscr{L}^{\infty}(V)\right)$ be a vertex, and $\Sigma_{s}$ be the open sub-complex of $\mathscr{L}^{\infty}(V)$ with only one vertex s. Then, there is a finite number of polyhedrons $P$ of dimension $k$, asymptotic to the amoeba $\mathscr{A}(V)$ in the direction of $s$, and such that $\overline{r(P)} \cap S^{n-1} \subset \Sigma_{s}$.

Proof. If the number of polyhedrons $P$ of dimension $k$, asymptotic to the amoeba $\mathscr{A}(V)$ in direction $s$, and such that $\overline{r(P)} \cap S^{n-1} \subset \Sigma_{s}$, is infinite, then there exists a sequence of parallel polyhedrons $\left\{P_{m}\right\}$ satisfying the same property. Hence, there are two possibilities: (i) the number of their corresponding $k$-dimensional real torus in $\left(S^{1}\right)^{n}$ is finite, which means that at least one of them is covered under the argument map infinitely many times. This contradict the fact that the volume of the amoeba is finite. (ii) the number of their corresponding $k$-dimensional real torus in $\left(S^{1}\right)^{n}$ is infinite, which means that they contain at least one accumulation $k$-torus (of course parallel to all of them). This is a contradiction with the assumption on the volume of the amoeba.

Let $V \subset\left(\mathbb{C}^{*}\right)^{n}$ be a generic $k$-dimensional analytic variety with defining ideal $\mathcal{I}(V)$ such that the volume of its amoeba is finite. Let $s$ be a vertex of $\mathscr{L}^{\infty}(V)$ with slope $\left(u_{1}, \ldots, u_{n}\right)$, and $\mathscr{P}_{s}$ be the finite set of polyhedrons in the direction of $s$, and asymptotic to the amoeba $\mathscr{A}(V)$. We denote by $\mathscr{H}\left(\mathscr{P}_{s}\right)$ the union of the holomorphic cylinders which are the lifting of the polyhedrons in $\mathscr{P}_{s}$, and asymptotic to the ends of $V$ corresponding to $s$.

Lemma 5.2. Let $V \subset\left(\mathbb{C}^{*}\right)^{n}$ be a generic $k$-dimensional analytic variety. Then, the ideal $\mathcal{I}(V)$ is generated by a set of holomorphic functions $\left\{f_{1}, \ldots, f_{q}\right\}$ such that for any $j=1, \ldots, q$, we have $f_{j}=h_{j} g_{j}$, where $h_{j}$ are holomorphic functions without zeros in a neighborhood of $\mathscr{H}\left(\mathscr{P}_{s}\right)$, and $g_{j}$ are entire functions where the terms of their power series have powers in the closed half space $\{\alpha \mid \alpha . u \leq 0\}$ where $\alpha . u=\sum_{i=1}^{n} \alpha_{i} . u_{i}$.

Proof. The proof of this lemma is similar to the proof of Lemma 4.4 . 
End of Theorem 1.1 proof. The second implication i.e., (ii) $\Rightarrow$ (i) is a consequence of Theorem 3.1. Without loss of generality, we may assume that $V$ is irreducible. So, (i) $\Rightarrow$ (ii) is a consequence of Proposition 5.1. Lemma 5.1. Lemma 5.2, and Lemma 4.3 .

\section{EXAMPLES}

1. Let $\mathscr{C}$ be the complex plane curve in $\left(\mathbb{C}^{*}\right)^{2}$ given by the zeros of the holomorphic function $f\left(z_{1}, z_{2}\right)=z_{2}-e^{z_{1}}$. Since the complex rank of $\operatorname{Jac}(f)$ is one, $\mathscr{C}$ is a Riemann surface. A parametrization of $\mathscr{C}$ is given by $t \in \mathbb{C}^{*} \mapsto$ $\left(t, e^{t}\right) \in\left(\mathbb{C}^{*}\right)^{2}$. The amoeba $\mathscr{A}(\mathscr{C})$ is the set of points in $\mathbb{R}^{2}$ delimited by the graphs of the two functions $x \mapsto \pm e^{x}$ (see Figure 1). The set of critical points of $\log$ restricted to $\mathscr{C}$ is $S=\left\{\left(x, e^{x}\right) \in\left(\mathbb{R}^{*}\right)^{2}\right\}$. The map Log is 2sheets covering over its regular values. However, the map Arg is not a locally finite covering (i.e., \# $\operatorname{Arg}^{-1} \theta=+\infty$ for any regular value $\theta \in \operatorname{co\mathscr {A}}$ ). Since the closure of the coamoeba is compact, its area is always finite. But, the amoeba has an infinite area. This means that the assumption on Theorem 3.1 is necessary.

Moreover, we can check that the logarithmic limit set of $\mathscr{C}$ has 1-dimensional connected component and one isolated point of rational slope. The phase limit set of $\mathscr{C}$ is the whole torus.

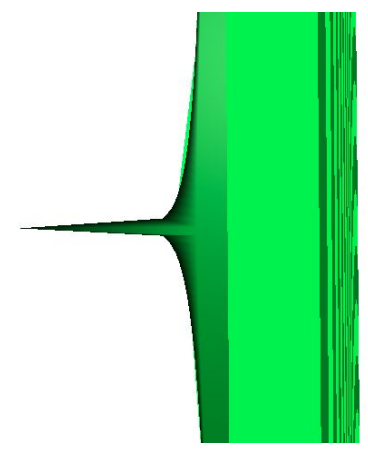

FIgURE 1. The amoeba of the plane holomorphic curve given by the parametrization $g(z)=\left(z, e^{z}\right)$.

2. Let $\mathscr{C}$ be the complex plane curve in $\left(\mathbb{C}^{*}\right)^{2}$ parametrized by

$$
\begin{aligned}
\rho: D & \longrightarrow\left(\mathbb{C}^{*}\right)^{2} \\
t & \longmapsto(\cos t, \sin t)
\end{aligned}
$$

where $D=\left\{t=a+i b \mid(a, b) \in(] 0,2 \pi\left[\backslash\left\{\frac{\pi}{2}, \pi, \frac{3 \pi}{2}\right\}\right) \times \mathbb{R}\right\}$ is the fundamental domain. We can check that $\mathscr{C}$ is contained in the algebraic curve with defining polynomial $p(x, y)=x^{2}+y^{2}-1$. On the other hand, we know that the last curve is irreducible. Hence, $\mathscr{C}$ is algebraic and defined by the same polynomial. The critical points of $\log$ are $\rho\left((] 0,2 \pi\left[\backslash\left\{\frac{\pi}{2}, \pi, \frac{3 \pi}{2}\right\}\right) \times\{0\}\right)$. The logarithmic limit set consists of three points with coordinates $(-1,0)$, $(0,-1)$ and $\left(\frac{\sqrt{2}}{2}, \frac{\sqrt{2}}{2}\right)$. The phase limit set consists of three pairs of circles with distinct slopes given by $-\infty, 0$ and 1 . 
3. Let $\mathscr{C}$ be the complex plane curve in $\left(\mathbb{C}^{*}\right)^{2}$ parametrized by

$$
\begin{aligned}
\rho: D & \longrightarrow\left(\mathbb{C}^{*}\right)^{2} \\
t & \longmapsto\left(z, e^{z}, z+1\right)
\end{aligned}
$$

The logarithmic limit set of $\mathscr{C}$ is the union of two points and an arc in the sphere $S^{2}$. Its phase limit set is an arrangement of two circles and 2dimensional flat torus. The curve $\mathscr{C}$ is not algebraic and the area of its amoeba (see Figure 2) is infinite.

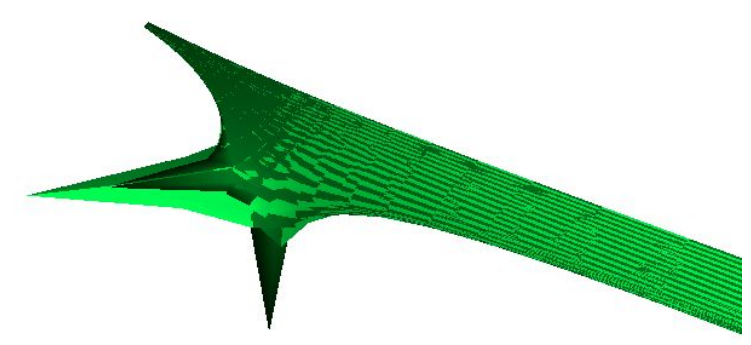

FiguRE 2. The amoeba of the spatial holomorphic curve given by the parametrization $g(z)=\left(z, e^{z}, z+1\right)$.

\section{Amoebas of $k$-Dimensional affine linear spaces}

Let $k$, and $s$ be two positives integers, and $\mathscr{P}(k) \subset\left(\mathbb{C}^{*}\right)^{k+s}$ be the affine linear space of dimension $k$ given by the parametrization $\rho$ :

$$
\begin{aligned}
\rho:\left(\mathbb{C}^{*}\right)^{k} & \longrightarrow\left(\mathbb{C}^{*}\right)^{k+s} \\
\left(t_{1}, \ldots, t_{k}\right) & \longmapsto\left(t_{1}, \ldots, t_{k}, f_{1}\left(t_{1}, \ldots, t_{k}\right), \ldots, f_{s}\left(t_{1}, \ldots, t_{k}\right)\right),
\end{aligned}
$$

such that $f_{j}\left(t_{1}, \ldots, t_{k}\right)=b_{j}+\sum_{i=1}^{k} a_{j i} t_{i}$ where $a_{j i}$ and $b_{j}$ are complex numbers for $i=1, \ldots, k$ and $j=1, \ldots, s$. First of all, we can assume that $f_{1}\left(t_{1}, \ldots, t_{k}\right)=1+\sum_{i=1}^{k} t_{i}$. Moreover, we assume that the affine linear spaces are in general position.

Definition 7.1. Let $V \subset\left(\mathbb{C}^{*}\right)^{n}$ be an algebraic subvariety, and conj be the involution on $\mathbb{C}^{n}$ given by conjugation on each coordinate. We say the variety $V$ is real if it is invariant under conj, i.e., $\operatorname{conj}(\mathrm{V})=\mathrm{V}$. The real part of $V$ denoted by $\mathbb{R} V$ is the set of points in $V$ fixed by conj.

In this section, we assume $n=2 k+m$, where $m$ is a nonnegative integer (i.e., $s=k+m$ ). The goal of this section is to prove the following theorem:

Theorem 7.1. Let $\mathscr{P}(k)$ be a generic linear space in $\left(\mathbb{C}^{*}\right)^{2 k+m}$, and $\theta$ is a regular value of the argument map. Then, the cardinality of $\operatorname{Arg}^{-1}(y)$ is equal to one. 
Before we give the proof of this theorem, let us start by looking to the case $k=1$, and the following remark:

Remark 7.1. Without loss of generality, we can assume that $\mathscr{P}(1)$ is the line in $\left(\mathbb{C}^{*}\right)^{2+m}$ given by the parametrization:

$$
\begin{aligned}
\rho: \mathbb{C}^{*} & \longrightarrow\left(\mathbb{C}^{*}\right)^{2+m} \\
t_{1} & \longmapsto\left(t_{1}, f_{1}\left(t_{1}\right), \ldots, f_{1+m}\left(t_{1}\right)\right),
\end{aligned}
$$

such that $f_{1}\left(t_{1}\right)=1+t_{1}$, and $f_{j}\left(t_{1}\right)=b_{j}+a_{j 1} t_{1}$, where $a_{j 1}$ and $b_{j}$ are complex numbers for $j=2, \ldots, 1+m$. Let $y=\left(y_{1}, \ldots, y_{2+m}\right)$ be a regular value of the logarithmic map. The number of point $t_{1} \in \mathbb{C}^{*}$ such that $\left|t_{1}\right|=e^{y_{1}}$, and $\left|f_{1}\left(t_{1}\right)\right|=e^{y_{2}}$ is at most two (because the intersection of two circles cannot exceed two points; the first circle has a center at the origin and radius $e^{y_{2}}$ and the second has the center at $(1,0)$ and radius $\left.e^{y_{1}}\right)$. It is clear that if $y$ is regular, then that number is equal two. Otherwise, the circles are tangent, and then $y$ is critical. Indeed, if we make a small perturbation of $t_{1}$ in some direction, the point goes out of the amoeba, which means that $y$ is in the boundary of the amoeba. Moreover, we can check that these two points are conjugate. So, if the number is equal two, then $\left|f_{j}\left(t_{1}\right)\right|$ should be equal to $\left|f_{j}\left(\bar{t}_{1}\right)\right|$ for any $j=1, \ldots, 1+m$. This means that $\left|b_{j}+a_{j 1} t_{1}\right|=\left|b_{j}+a_{j 1} \bar{t}_{1}\right|$, or equivalently $\cos \left(\arg \left(a_{j 1}\right)+\arg \left(t_{1}\right)-\arg \left(b_{j}\right)\right)=\cos \left(\arg \left(a_{j 1}\right)+2 \pi-\arg \left(t_{1}\right)-\right.$ $\left.\arg \left(b_{j}\right)\right)$. Hence, $\arg \left(a_{j 1}\right)-\arg \left(b_{j}\right)=0 \bmod (\pi)$, which is equivalent to $\frac{a_{j 1}}{b_{j}} \in \mathbb{R}^{*}$ for any $j$, and then the curve is real. Otherwise, the cardinality of $\log ^{-1}(y)$ is equal one.

Proof of Theorem 7.1. Let $\theta=\left(\theta_{1}, \ldots, \theta_{k}, \psi_{1}, \ldots, \psi_{k+m}\right)$ be a regular value of the argument map. If $\rho(t) \in \mathscr{P}(k)$ belongs to the inverse image by the argument map of $\theta$, then $\arg \left(t_{j}\right)=\theta_{j}$ for each $j=1, \ldots, k$, and $\arg \left(f_{l}(t)\right)=$ $\psi_{l}$ for each $l=1, \ldots, k+m$. The $k$-plane $\mathscr{P}(k)$ is parametrized by $\rho$ as above. For each $l$, we can always view $f_{l}, b_{l}$, and the $a_{l j} t_{j}$ 's as vectors in the plane $\mathbb{C}$, such that their arguments are in the increasing order. If we put them in the plane with this order, we obtain a convex polygon. We can check that if there exist $t \neq t^{\prime}$ with $\rho(t)$ and $\rho\left(t^{\prime}\right)$ in $\mathscr{P}(k)$, and $\operatorname{Arg}(\rho(t))=\operatorname{Arg}\left(\rho\left(t^{\prime}\right)\right)=\theta$, then the inverse image of $\theta$ by Arg has dimension strictly greater than zero. Therefore, $\theta$ in not regular. In fact, if $\left|t_{j}\right| \neq\left|t_{j}^{\prime}\right|$ for some $j$, then for any $\left(\lambda\left|t_{j}\right|+(1-\lambda)\left|t_{j}^{\prime}\right|\right) e^{\theta_{j}}$, with $\lambda \in[0,1]$, there exist $t_{s}=\mu_{s} e^{\theta_{s}}$ with $\mu_{s} \in \mathbb{R}_{+}$, $s \in\{1, \ldots, k\} \backslash\{j\}$, and $\operatorname{Arg}\left(f_{l}\right)=\psi_{l}$ for each $l=1, \ldots, k+m$. This means that the dimension of $\operatorname{Arg}^{-1}(\theta)$ is at least one. We conclude that the cardinality of $\operatorname{Arg}^{-1}(\theta)$ is equal one for any regular point $\theta$.

In the case of $k$-dimensional real linear spaces of $\left(\mathbb{C}^{*}\right)^{2 k}$, the second author, Johansson and Passare [JNP-10] prove that each regular value of the logarithmic map is covered by $2^{k}$ points, and the volume of the coamoeba is equal to $\pi^{2 k}$. Hence, the numbers $p$ and $P$ of Theorem 3.1 are equal to $\frac{1}{2^{k}}$ in this case. 
Corollary 7.1. Let $\mathscr{A}(k)$ be the amoeba of a $k$-dimensional real linear space of $\left(\mathbb{C}^{*}\right)^{2 k}$. Then,

$$
\operatorname{vol}(\mathscr{A})=\frac{\pi^{2 k}}{2^{k}}
$$

\section{REFERENCES}

[B-71] G. M. Bergman, The logarithmic limit-set of an algebraic variety, Trans. Amer. Math. Soc. 157, (1971), 459-469.

[BG-84] R. Bieri AND J.R.J. Groves, The geometry of the set of characters induced by valuations, J. Reine Angew. Math. 347, (1984), 168-195.

[FPT-00] M. Forsberg, M. Passare And A. Tsikh, Laurent determinants and arrangements of hyperplane amoebas, Advances in Math. 151, (2000), 45-70.

[GKZ-94] I. M. Gelfand, M. M. Kapranov and A. V. Zelevinski, Discriminants, resultants and multidimensional determinants, Birkhäuser Boston 1994.

[H-03] A. Henriques, An analogue of convexity for complements of amoebas of varieties of higher codimensions, Advances in Geometry, Adv. Geom. 4, (2003), 61-73.

[JNP-10] P. Johansson, M. Nisse And M. Passare, (Co)Amoebas of linear spaces, in preparation.

[MN-11] F. MAdAni, M. Nisse, On the volume of complex amoebas, preprint, http://arxiv.org/pdf/1101.4693.

[M1-02] G. Mikhalkin, Decomposition into pairs-of-pants for complex algebraic hypersurfaces,Topology 43, (2004), 1035-1065.

[M2-04] G. Mikhalkin, Enumerative Tropical Algebraic Geometry In $\mathbb{R}^{2}$, J. Amer. Math. Soc. 18, (2005), 313-377.

[M3-00] G. Mikhalkin , Real algebraic curves, moment map and amoebas, Ann.of Math. 151 (2000), 309-326.

[NS-09] M. Nisse AND F. SotTile, The phase limit set of a variety, preprint, http://arxiv.org/pdf/1106.0096

[NS-11] M. Nisse AND F. SotTILE, Non-Archimedean coAmoebae, in preparation.

[PR-04] M. PAssare And H. RullgÅrd, Amoebas, Monge-Ampère measures, and triangulations of the Newton polytope, Duke Math. J. 121, (2004), 481-507.

[PS-04] L. Pachter and B. Sturmfels, Algebraic Statistics for Computational Biology, Cambridge University Press, 2004.

NWF I-Mathematik, Universität Regensburg, D-93040 Regensburg, Germany. E-mail address: Farid.Madani@mathematik.uni-regensburg.de

Department of Mathematics, Texas A\&M University, College Station, TX 77843-3368, USA.

E-mail address: nisse@math.tamu.edu 